\title{
Myeloid Neoplasms with Eosinophilia: Rare Entities with Emerging Diagnostic and Therapeutic Challenges
}

\author{
Giulia Benevolo Irene Urbino \\ Division of Haematology, Città della Salute e della Scienza, Turin, Italy
}

Hypereosinophilia-HE (a persistent eosinophil count of $>1.5 \times 10^{9} / \mathrm{L}$ in peripheral blood or a higher than $20 \%$ of eosinophils in bone marrow section) may be observed in many reactive and clonal disorders, and it may be associated with life-threatening organ damage as a result of tissue infiltration and release of granular contents [1]. In patients presenting with hypereosinophilia, an initial workup to rule out reactive causes should be made, considering atopic conditions, infections, medications, autoimmune disorders, and hematological or solid malignancies. If no secondary causes are found, a primary eosinophilia should be suspected. Evaluation includes morphologic analysis of the peripheral blood and bone marrow, immunohistochemistry (CD117, tryptase, and CD25), flow cytometric immunophenotyping to assess the presence of myeloid or B/T-lymphocyte markers, and cytogenetic/molecular/genetic testing $[1,2]$. According to revised 2016 World Health Organization classification, it is recommended to test for FIP1L1-PDGFRA by FISH or RT-PCR and to performed cytogenetic/FISH analysis for 4q12(PDGFRA), 5q31 q33(PDGFRB), 8p11 12 (FGFR1), or 9p24 (JAK2) [1, 2]. The most frequent JAK2 fusion gene is PCM1-JAK2 as a consequence of a $t(8 ; 9)$ (p22;p24). The translocation $(8 ; 9)$ is diagnosed by dualcolor FISH showing a fusion signal, and the fusion transcript is confirmed by RT-PCR. Cytogenetic analysis

\footnotetext{
karger@karger.com

(c) 2020 S. Karger AG, Basel

www.karger.com/aha

Karger
}

showed that the break points are various, involving exons 23-36 of PCM1 and exons 1-11 of JAK2. The PCM1JAK2 fusion gene is predicted to encode a protein of 257$310 \mathrm{kDa}$, depending on the positions of the break points [1]. Patients with PCM1-JAK2 usually present with features of a chronic myeloid neoplasm (MPN) and are frequently associated with eosinophilia and/or bone marrow fibrosis [1]. The clinical course is aggressive, with rapid progression from chronic phase disease to AML, and more rarely to lymphoid blast phase. Patients may also present with de novo acute leukemia [1].

In this issue of Acta Haematologica, Cai et al. [3] describe a patient diagnosed with this rare eosinophilia-associated myeloproliferative neoplasm, involving exon 33 of PCM1 and exon 11 of JAK2. The patient is a young man referring to the hospital for progressive knee pain, with laboratory tests showing leukocytosis, eosinophilia, anemia, splenomegaly, and bone marrow fibrosis.

Since up to $60 \%$ of MPN with PCM1-JAK2 are accompanied by eosinophilia, evaluation for $\mathrm{t}(8 ; 9) / \mathrm{PCM} 1-\mathrm{JAK} 2$ is highly recommended for all patients presenting with myeloid neoplasm and a persistent high eosinophil count.

Editorial comment on the paper by Cai et al.: Sustained response to ruxolitinib of eosinophilia-associated myeloproliferative neoplasm with translocation t(8;9)(p21;p24), Acta Haematol. 
However, the diagnosis may be difficult and requires a range of analysis that are not always readily available, as described by Cai et al. [3] who were able to diagnose $t(8 ; 9)$ (p21;p24) only few months after the onset of the disease, when it became more aggressive.

Overall, many therapeutic regimens have been attempted to treat these conditions with only short-term clinical remissions achieved. Therefore, at present, the only potentially curative option for these patients is the allogeneic stem cell transplantation (HSCT) $[1,4,5]$. In the largest case series, allogeneic HSCT was performed in 5 of 13 younger than 55-year patients with PCM1-JAK2 fusions, either in chronic phase or in first remission after intensive chemotherapy of acute leukemia, showing a progression-free survival of 33 months [1]. To handle myeloproliferative neoplasms with $t(8 ; 9)$ in nontransplant eligible patients, the main treatments used before the advent of JAK2-kinase inhibitors were hydroxyurea and interferon alpha (INF $\alpha$ ), but no long remissions were achieved, with an overall survival $<12$ months $[1,4,5]$.

Since its appearance, JAK2-inhibitor ruxolitinib has changed therapeutic approach to MPN becoming a relevant option also for patients carrying $\mathrm{t}(8 ; 9)$ translocation [4]. In young patients, it might play a role as bridging therapy to transplant, but its role in not eligible transplant patients is still ambiguous and needs more evidence [5].

Among the 42 reported cases with $\mathrm{t}(8 ; 9) / \mathrm{PCM} 1-\mathrm{JAK} 2$, only 3 patients presenting with a MDS/MPN received ruxolitinib treatment, one of which relapsed after 18 months and required a subsequent allogeneic transplant $[3,5]$. The median survival time for the other 2 patients was 26 months [5]. The first of them was a 72 -year-old man with chronic eosinophilic leukemia, treated with ruxolitinib at a dose up to $20 \mathrm{mg}$ twice per day, who achieved a complete cytogenetic response after 15 months from diagnosis and was alive at 30 months $[1,4]$. The other one was a 31-year-old woman with PCM1-JAK2 positive myeloid neoplasm, lacking a compatible stemcell donor, who was treated with a dose of $15 \mathrm{mg}$ twice per day of ruxolitinib and obtained a complete clinical remission with regression of anemia, leukocytosis, eosinophilia, splenomegaly, and marrow fibrosis, improvement of thrombocytopenia, and restoration of polyclonal hematopoiesis at 12 months after start of therapy [4].

The patient described by Cai et al. [3] had a relatively satisfactory outcome after ruxolitinib monotherapy, with good clinical response and complete hematological remission at 17 months from the beginning of treatment. Furthermore, the drug was well tolerated without significant adverse events. However, despite encouraging preliminary data, long-term response of ruxolitinib remains unclear, particularly considering that no molecular remissions have been reported.

In conclusion, due to the low incidence of the disease, at the moment there is no consensus about therapeutic strategy and approved response criteria for the treatment of patients affected by MDS/MPN with PCM1-JAK2 fusion gene. However, it seems that we are entering a time of new therapeutic hopes and challenges for these patients, even for those not eligible for HSCT. Thus, data addressing this issue are both welcomed and needed.

\section{Conflict of Interest Statement}

Dr. Benevolo received honoraria from Novartis, Celgene, Amgen. Dr. Urbino has no conflicts of interest to declare.

\section{Author Contributions}

Both authors contributed to the writing of this editorial.

\section{References}

1 Reiter A, Gotlib J. Myeloid neoplasms with eosinophilia. Blood. 2017 Feb 9;129(6):70414.

2 Arber DA, Orazi A, Hasserjian R, Thiele J, Borowitz MJ, Le Beau MM, et al. The 2016 revision to the World Health Organization classification of myeloid neoplasms and acute leukemia. Blood. 2016 May 19;127(20):2391405.
3 Cai P, Liu S, Duan L, Huo L, Wu D, Chen S, et al. Sustained response to ruxolitinib of eosinophilia-associated myeloproliferative neoplasm with translocation $\mathrm{t}(8 ; 9)(\mathrm{p} 21 ; \mathrm{p} 24)$. Acta Haematol. DOI: 10.1159/000510281.

4 Rumi E, Milosevic JD, Casetti I, Dambruoso I, Pietra D, Boveri E, et al. Efficacy of ruxolitinib in chronic eosinophilic leukemia associated with a PCM1-JAK2 fusion gene. J Clin Oncol. 2013 Jun 10;31(17):e269-71.
5 Schwaab J, Knut M, Haferlach C, Metzgeroth G, Horny HP, Chase A, et al. Limited duration of complete remission on ruxolitinib in myeloid neoplasms with PCM1-JAK2 and BCRJAK2 fusion genes. Ann Hematol. 2015 Feb; 94(2):233-8. 